\title{
Efficient connectivity in Wireless Sensor Network using adaptive strategy in evolutionary computation
}

\author{
Channakrishnaraju ${ }^{\# 1}$, M.Siddappa ${ }^{* 2}$, \\ ${ }^{\# 1}$ Research Scholar, Sri Siddhartha Academy of Higher Education [SAHE], Tumkur, India \\ ckrajussit@gmail.com. \\ ${ }^{* 2}$ Prof. \& Head, Dept. of Computer Science \& Egg. Sri Siddhartha Institute of Technology, Tumkur, India \\ siddappa.p @gmail.com
}

\begin{abstract}
At present, wireless sensor network (WSN) has reeived intense attention by reserchers because of its utilities in the various applications.In WSN ,sensors are battery operated devices and most of cases practicaly it is not possible to replace the battery once it loose the life.There are various reasons for energy consumption and among them one very significant factor is unefficient connectivity of sensors for sharing the relevent information under network.Connectivity situation can be more worse if there is a dynamic environment exist under network environment.In this paper ,evolutionary approach based on various form of genetic algorithm has proposed to handle this issue.Different strategy like, redifiniton of agents,inclusion of flying agents and carrying the experience have included to enhance the qulaity of solution.
\end{abstract}

Keyword- wireless sensor network, connectivity, energy efficiency, evolutionary computation

\section{INTRODUCTION}

A wireless sensor network (WSN) is uses wireless communication to obtain information by using tiny electronic devices called sensors, which is used in remote area such as thick forest, furnace area and area in which human beings cannot stay for long time. These sensors are located at different locations in the area in which gather information and send to the main station or base station. A wireless sensor network (WSN) has significant merits such as gathering the information in isolated ecological observations and tracking the desired targets located in different places. The energy source of a sensor node is a battery cell, and they can sojourn vigorous for a restricted time before the energy of the battery is exhausted. The battery is to be recharged very often to make the battery in active mode, it is not advisable in many tasks. So the energy of node is to be preserved by longer duration. The coverage of targets with minimum energy is a research problem problem in WSNs. Recent advances in Micro Electronics and wireless technology have highlighted the significance of WSNs as essential reporting devices. Indeed, sensor nodes in WSNs are deemed to be resource constrained in terms of energy, communication range, and memory capacity and processing capability. The important objective of WSNs is to disseminate the data from the source to the destination node in multi-hop scheme. In WSNs, transmission and reception of data packets are considered as the chief sources of energy consumption.Hence it is necessary that connectivity between source and sink has to be defined through minimum number of hopes which include the minimum distance as much as possible.In many applications,topology if network is keep changing because of various reasons like new connectivity inclusion or deletion of existing ones.It is very necessary,not only to provide unintrupted connectivity between source and sink with new topology of network as soon as possible but also connectivity should be energy efficient.Simulation modeling of WSN can be defined through the help of graph theory notation and terminology

\section{RELATED WORK}

Because of huge applications, number of researcher have applied the research in the area of WSN and related aspects.[1] has specified the outline of some recent applications and then analyze the different problems like deployment of nodes, provisioning and different network services in WSNs. The efficient node deployment method with minimum cost, moderate reckoning and message transformation overhead, be robust to node ruins is implemented. Implementation of efficient method for better coverage and connectivity of wireless sensor networks is awfully perplexing. Therefore efficient coverage with optimized network connectivity between the nodes is a critical problem. In [2], authors have presented and provide the comparison between different algorithms and methods to overcome the coverage-connectivity problem. The placement strategy has discussed in [3] as implementation of better method for achieving the anticipated design goals with minimum data latency. The energy depletion for the parts of a typical sensor node has presented in [4], and discussed the energy saving in WSNs. They have presented an efficient energy saving schemes. The authors are focused on different methods for energy efficient data gathering. The efficient node deployment method in wireless sensor networks has discussed in [5] to provide the efficient network topology with minimum cost of deployment, better 
connectivity of networks and elevating network lifespan. The NP-complete problem is articulated by using combinatorial optimization method. They have suggested potential field deployment algorithm (PFDA) and multi-objective deployment algorithm (MODA). [6]This paper analyzes the metric relationship model with 3-D Clifford sensor network. The Clifford sensor network contains connection graph with independent of coordinate and is reliable with different targets in diverse dimensional space.[7] The authors planned a deliberate model with queuing to obtain an optimum solution to elevate energy depletion of the sensor node. [8] Has investigated the connectivity of random deployment nodes in WSN with Gaussian distribution. This method provides better results while simulating in nonlinear distribution of sensors. The important problem in WSNs is Coverage and connectivity problems, which have a abundant effect on the performance of WSNs. The Improved method with efficient node deployment approach is used. In [9], authors have categorized the problem of coverage in WSN with different angles, define the estimated metrics by using the suitable algorithms. In[10],the node deployment problem is articulated with multi-objective optimization (MO) problem where, the objective of the problem is to discover a deployed sensor node to get the best out of coverage of targets, minimum the network energy depletion, better network lifespan, and connectivity between source and destination node for accurate transmission of information with minimum number of sensor nodes. [11] Presented the essential study on the connectivity between nodes in wireless sensor networks and efficient coverage of targets is obtained from mathematical modeling, theoretical study, and performance assessment perceptions. [12] In this paper the authors are presented, node deployment pattern with polygon shape to obtain optimum position of sensors in Wireless Sensor Networks for efficient coverage and connectivity. The important objective of this problem is to provide efficient connectivity and maximize the coverage with minimum number of sensors. To obtain better solution with minimum computational sources for node deployment problem with NP -hard is perplexing problem of research in WSN.[13] has presented an outline of WSN and node deployment problem in wireless sensor networks , and deliberations on metaheuristics and demonstrates how to use the meta heuristics methods to resolve the node Deployment Problem in WSN. The efficient method is used for curtail by using Sleep Scheduling (SS) mechanism and enhance lifespan of wireless sensor networks. In [14], authors presented software based algorithm to cope the energy of the wireless sensor network with Sleep Scheduling of nodes. In [15], review has presented the issues built on archetypal of WSNs: structured and non-structured for data gathering and aggregation and also discussed the importance of clustering and routing in wireless sensor networks for better energy preservation and lifespan of the network. In [16] authors have presented a distributed Resource Constrained Recovery method is used to restructured a network subdivided into dismember segments by deliberately relocation of nodes. The cases in which relocation nodes are inadequate to form steady topology of inter segment then mobile data gatherers with elevated routes to reduce data delay

\section{PROPOSED SOLUTON}

To obtain minimum distance, we have implemented adaptive genetic algorithms like, Redefined agents genetic algorithm [RAGA], Flying agents genetic algorithms[FAGA], Experienced agents genetic algorithms [EAGA].These algorithms provide better connectivity in dynamic environments of wireless sensor networks. The dynamic environments obtain when the sensors are moved from place to another place with the help of animals, robotics and human beings (soldiers). The adaptive genetic algorithms provide better solutions when the sensors in dynamic environment.

In WSN there is a number of applications where dynamic topology exists for example in the case of a) Track animals b) Soldier strategy in war field etc. To handle Dynamic wireless sensor networks there is requirement of high level adaptability. In natural system evolutions can we consider as best example for adoptability, hence genetic algorithm platform has adapted, but the existing challenges are

To find the optimal connectivity from one sensor to another for communication

Detect change in topology

With the change topology as shown as possible reestablish the optimal connectivity.

To handle all these three different approaches are developed as shown in the Fig-1.

(i)Apply the new agents from the beginning itself

(ii)Keep up line the flying agents continuously

(iii)The experienced agents accommodate in the next generations 


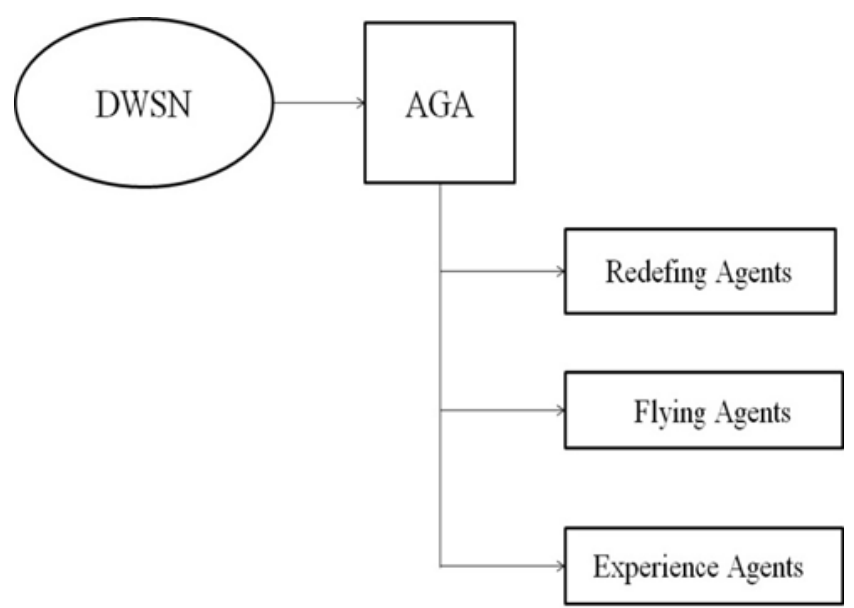

Fig1. Dynamic Environmental Connectivity

3.1Network development

To simulate the wireless sensor networks a defined range of area can be generated by random number of $\mathrm{x}$ co-ordinate and y co-ordinate through uniform distribution depends upon individual Euclidian distance on the communication range connectivity between sensors defined as shown in Fig-2

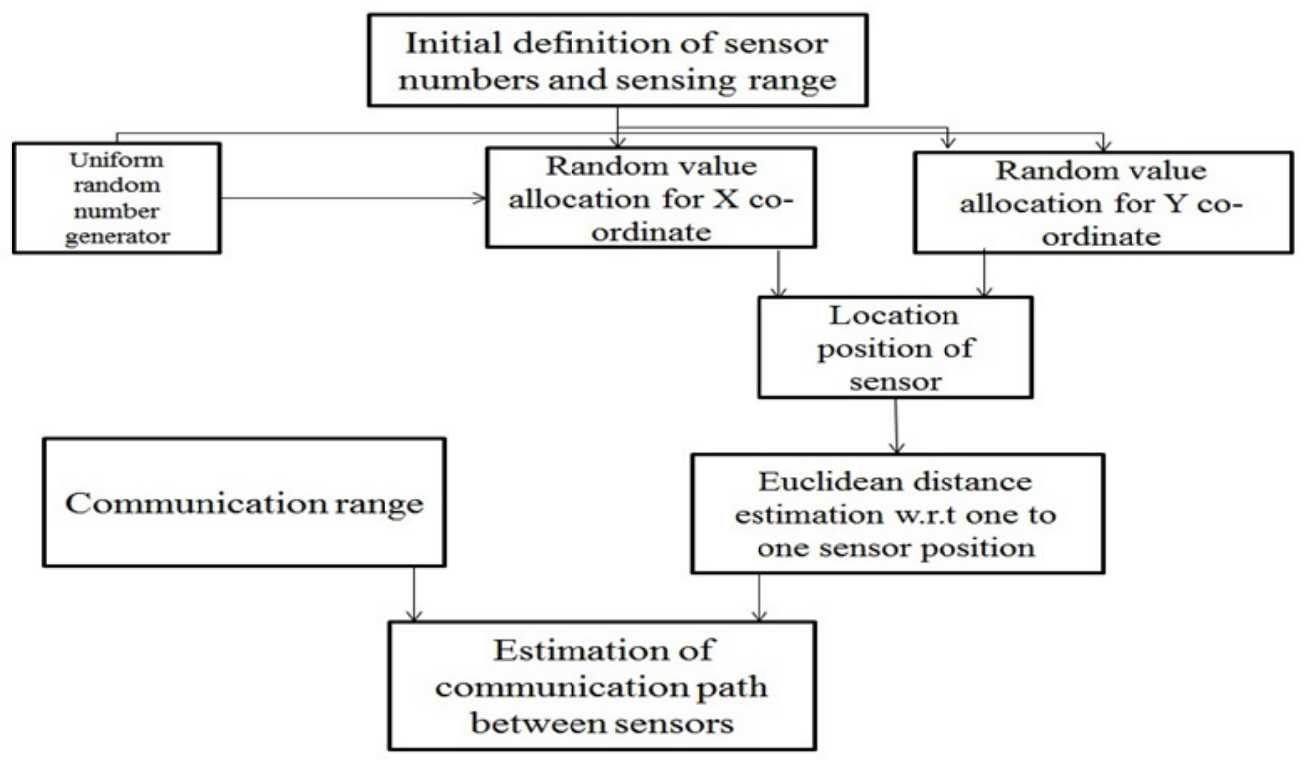

Fig 2. Flow chart of network development

\subsection{Redefined agents with time :( RAGA)}

Concept is handling the dynamic problem on a new start to solve this with this approach, based on redefining new agents which are fresh population to handle the problem from the beginning there is nearby guaranty to get optimal solutions because it does not have any pervious burden.With intial population definite genetic operator corss over and mutation applied to create offspring . The parent and offspring population are combined to get the new population through selection poeration. The dynamic condition of a network is established through getting stable change in the fitness value with the comparision the topology change can be decide if it is yes the new population and redifined agents will replace previous population to handle the appered topology and process is go on.The psudo code has given below. 
Psudo code for RAGA

1. Initialization of Population

2. Apply crossover and mutation operator to produce offsprings.

3. Mixer population [MPOP] $\leftarrow$ [Parent Pop; Offspring Pop]

4. Fitness estimation 『(f】_(vi )) $\leftarrow$ F_fun (MPOP)

5. Next Generation population $\leftarrow$ Tournament selecetion 『(f】_(vi ))

5. Topology change detection $\leftarrow$ f(f_vi-f_(v(i-1)) ) ;

6. If change detected

Current Generation = New Random Agent population ;

Else

Current Generation = Next Generation;

7. Go to step 1.

3.3 Flying agent's with time [FAGA]:

The new member are always incorporated with the populations in result there is a better diversity available and if there is dynamic condition appeared this diversity will help to find out solutions . With this approach it is possible to handle dynamic conditions faster.With initial definition of population the genetic operator applied to create offspring population and through tournament selection new population is created .The fitness value estimated and weak one is going to replace by newly created flying agents and this population will replace population and process is goes on. The psudo code for FAGA has given below.

Psudo code for FAGA

1. Initialization of Population

2. Apply crossover and mutation operator to produce offsprings.

3. Mixer population [MPOP] $\leftarrow$ [Parent Pop; Offspring Pop]

4. Fitness estimation 『(f $\left.\_\_(v i)\right) \leftarrow$ F_fun (MPOP)

5. Next Generation population [NPOP] $\leftarrow$ Tournament selecetion 『(f】_(vi ))

5. Replacement of Weak agents by Flying Agents from NPOP

6. Go to step 1 .

\subsection{Experienced agent's with time: [ EAGA]}

If there is possibility to place experienced agents in the next generation always there is very good chance to handle dynamic topology in efficient manner because they are having the knowledge to handle the change in the topology with the time in result the optimal solutions can be achieved with very less time and chances failure is minimum.In EAGA with initial population definition and applying the genetic operator through selection process new population is created among the new population whichever is having higher fitness value that is solution stored in memory. This process will goes on in every generation .The previously stored experienced solution will replace the weak solution available in current population to create find new population and process will continue. The psudo code has given below.

Fundamentally the two different approaches has applied to handle dynamic available in topology-

(i) Detect and change in topology and take action

(ii) Be adaptive all times, if there any change occur in topology it will handle by the same process

RAGA concept applied to handle detedct and change approach while FAGA and EAGA applied to be adaptive always.

Psudo code for EAGA

1. Initialization of Population

2. Apply crossover and mutation operator to produce offsprings.

3. Mixer population [MPOP] $\leftarrow$ [Parent Pop; Offspring Pop]

4. Fitness estimation 『(f】_(vi )) $\leftarrow$ F_fun (MPOP)

5. Next Generation population $[\mathrm{NPOP}] \leftarrow$ Tournament selecetion 『(f】_(vi ))

5. Store the best members from NPOP in $\square \mathrm{SB} \square_{\_} \mathrm{i}$

6. Replace the weak agent from NPOP by agents from $\llbracket \mathrm{SB} \rrbracket+(\mathrm{i}-1)$

6. Go to step 1. 


\subsection{Numerical Optimization}

\section{EXPERIMENTAL RESULTS}

Two understand the comparative benefit with proposed method, dynamic weight based PSO has applied to minimze over a multimodel function as given in Eq.1, whose complexity can understand by looking the landscpe defined by Eq.1 as shown in Fig.3 .FAGA and EAGA have also been applied and performances have shown in Fig.4 .It is clear that both FAGA and EAGA have performed better than DWPSO, but EAGA has shown the superior performance.

$\mathrm{F}=20+\mathrm{x} 1 . \wedge 2+\mathrm{x} 2 . \wedge 2-10 *(\cos (2 * \mathrm{pi} * \mathrm{x} 1)+\cos (2 * \mathrm{pi} * \mathrm{x} 2)) \quad-(1)$

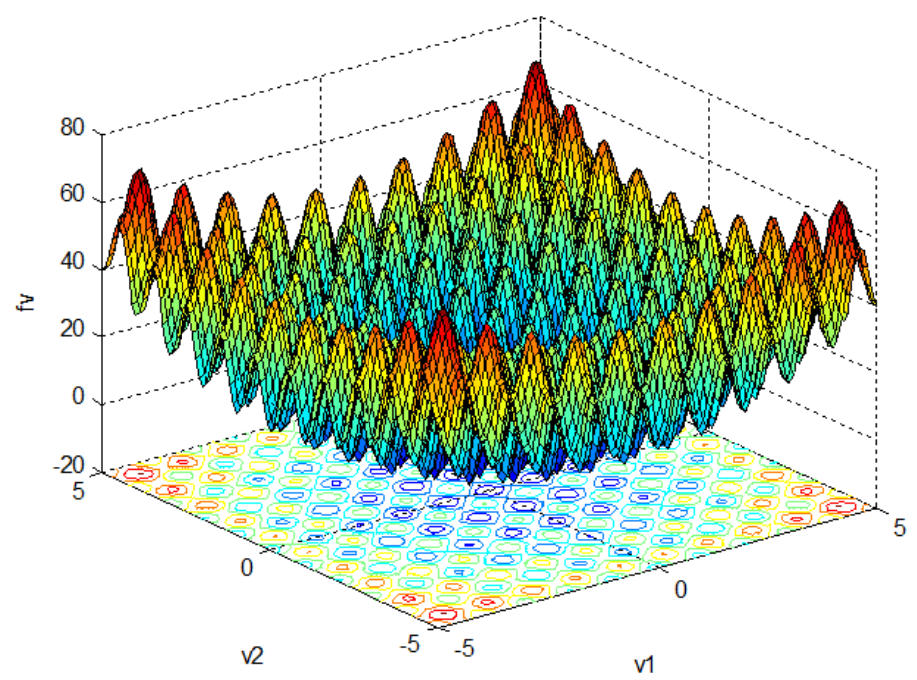

Fig.3 Three dimension plot of function F

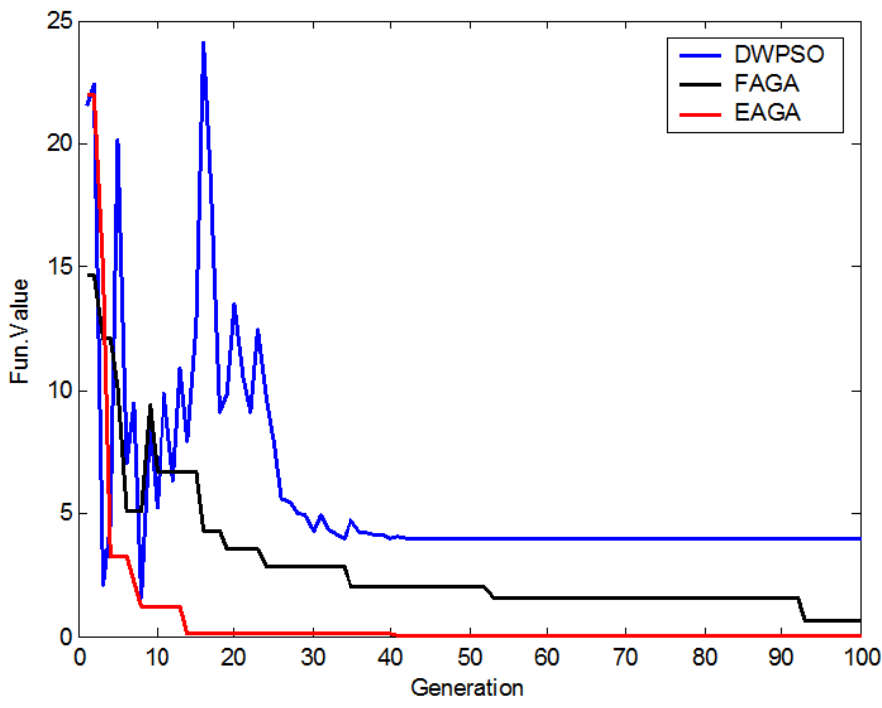

Fig.4. Perfromances over function

\subsection{WSN Connectivity}

Simulation experiment has done in MATLAB environment.Three different set of sensors 50,100 and 150 have applied in independent experiment and obtained results have shown in Table-1 and performance characteristics have represented in Fig.-5 to Fig.-14. For all the experimental case size of population is taken as 50.Crossover has applied with 1 probability if it is feasible else 0 .Mutation has applied with 0.1 probabilities. 
Experiment with Dropped Sensor Number: 50

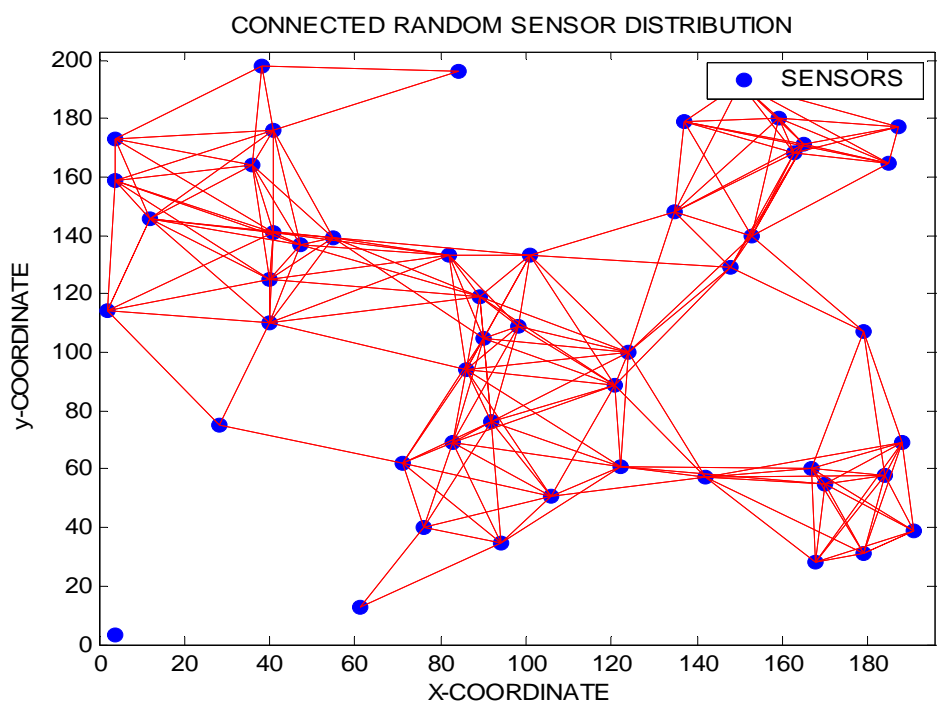

Fig-5 Connectivity of sensors

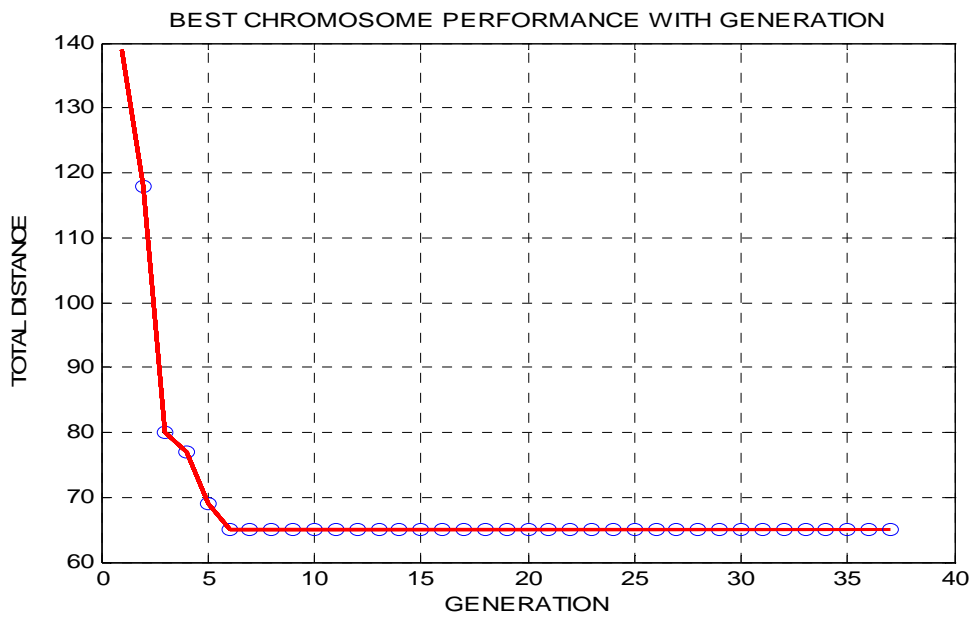

Fig-6 Static Connectivity path between 5[S] to 25[D] : [5, 47, 38, 22,25]

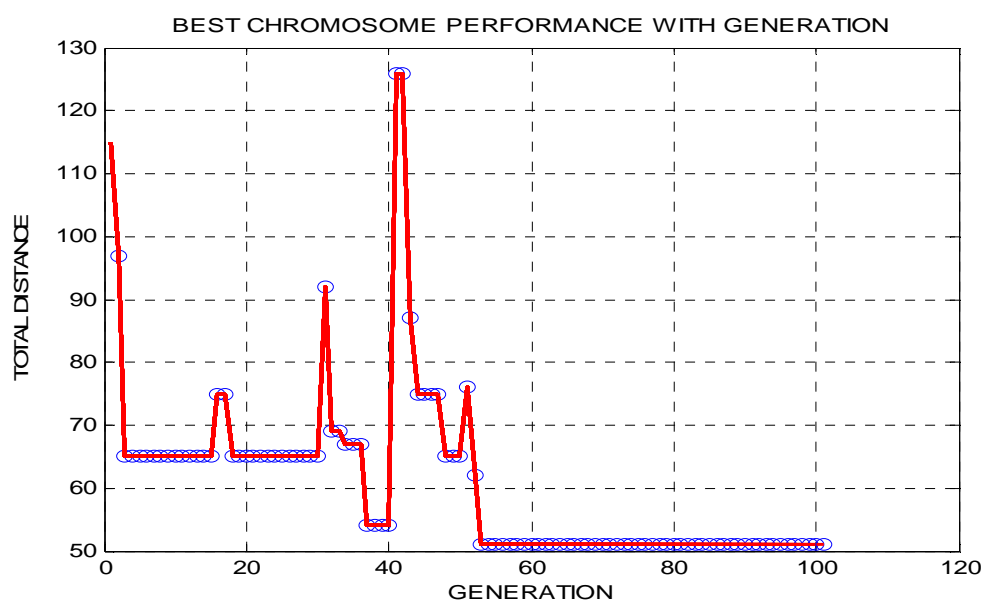

Fig-7 Redefined adoptive genetic algorithm ,Connectivity path between 5[S] to 25[D] : [5 47287 25] 


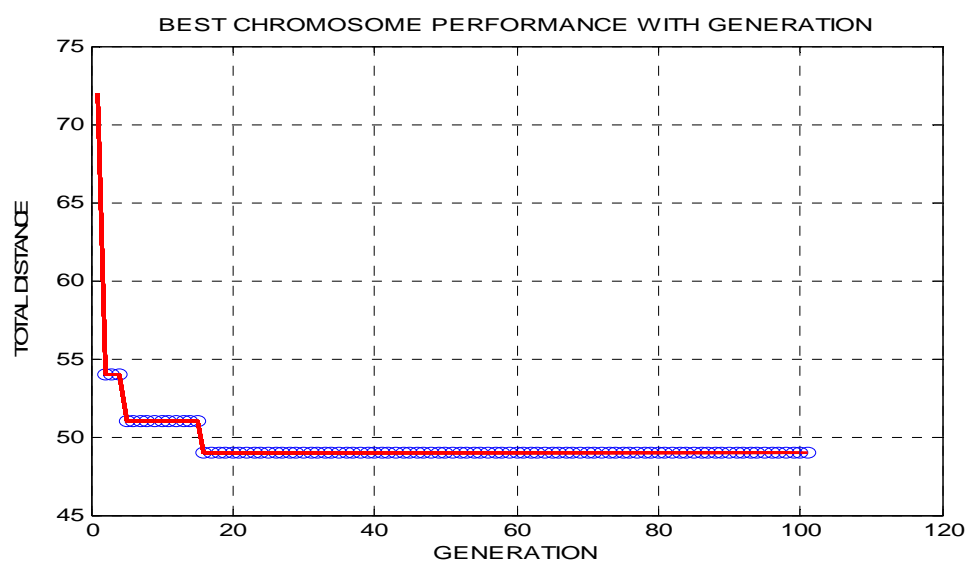

Fig-8: Flying agents adoptive genetic algorithm, Connectivity path between 5[S] to 25[D] : [5 1827 25]

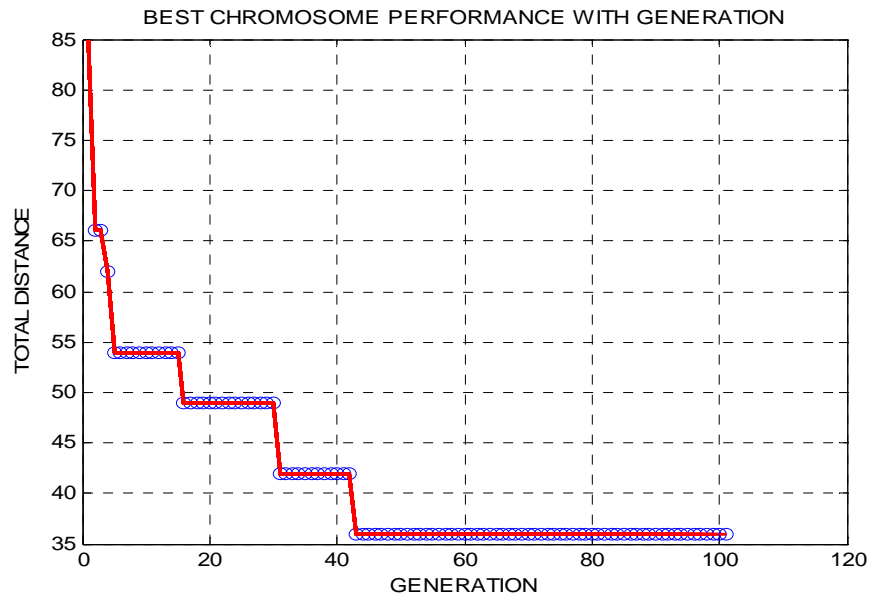

Fig-9 Experience agent adoptive genetic algorithm Connectivity path between 5[S] to 25[D] : [5 473 25]

Experiment with Dropped Sensor Number: 150

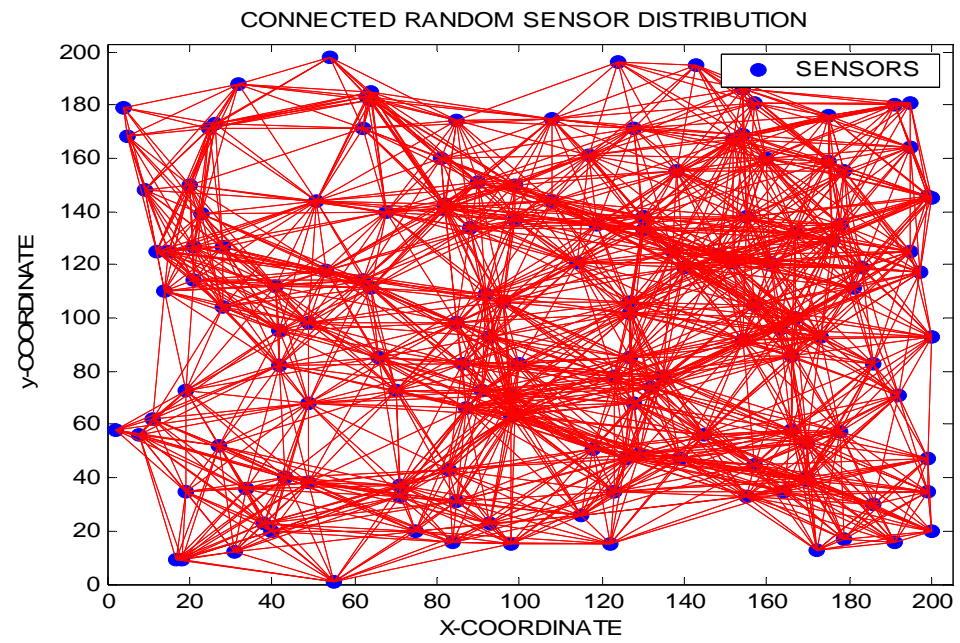

Fig-10 Connectivity of sensors 


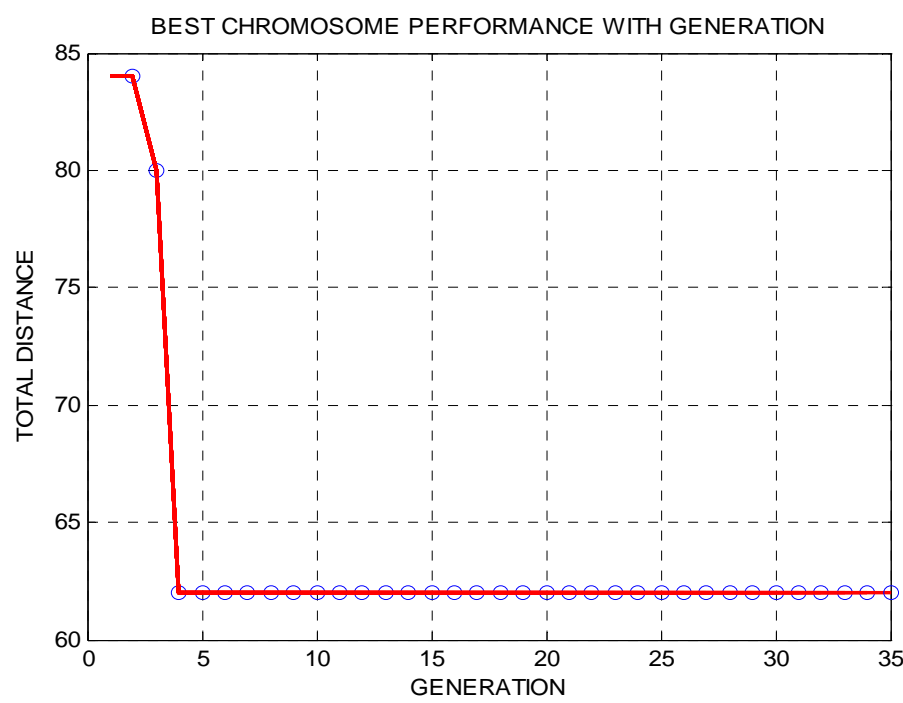

Fig-11 Static Connectivity path between 20[S] to 50[D] : [20 76,34 7550 ]

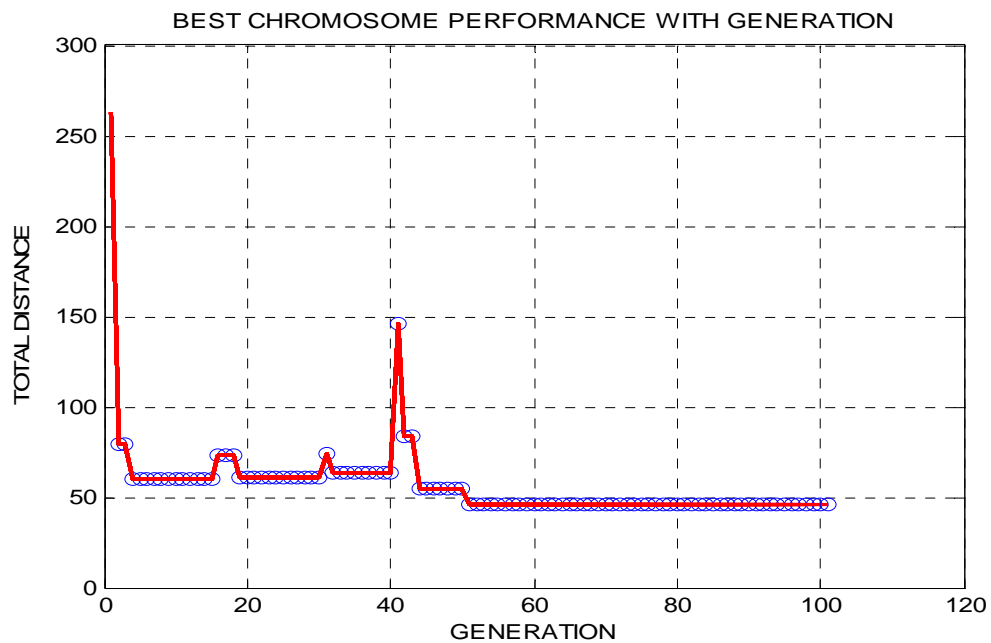

Fig-12 Redefined adoptive genetic algorithm Connectivity path between 20[S] to 50[D] :[20 9913 50]

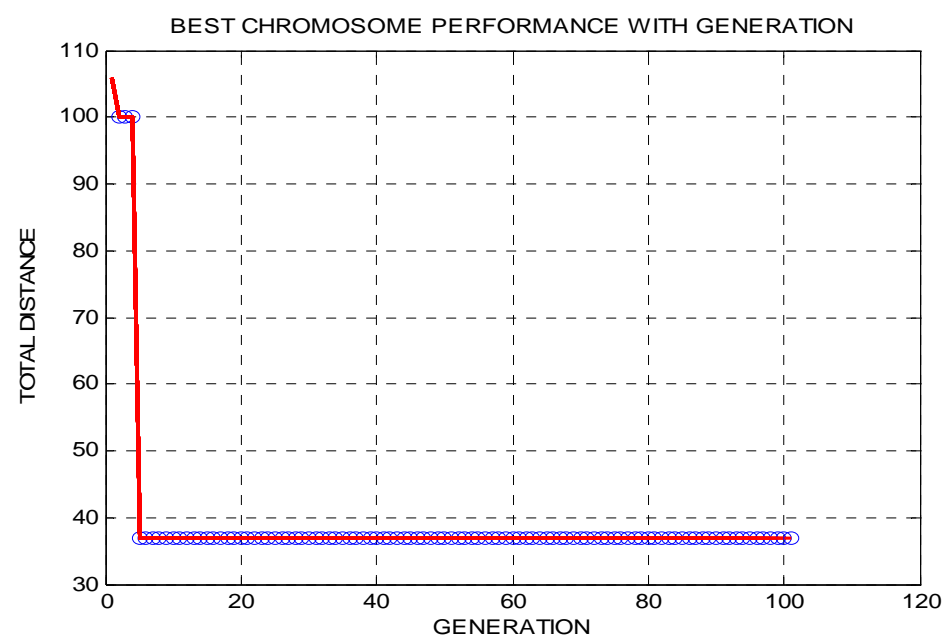

Fig-13 Flying agents adoptive genetic algorithm Connectivity path between 20[S] to 50[D] : [20 12614450 ] 


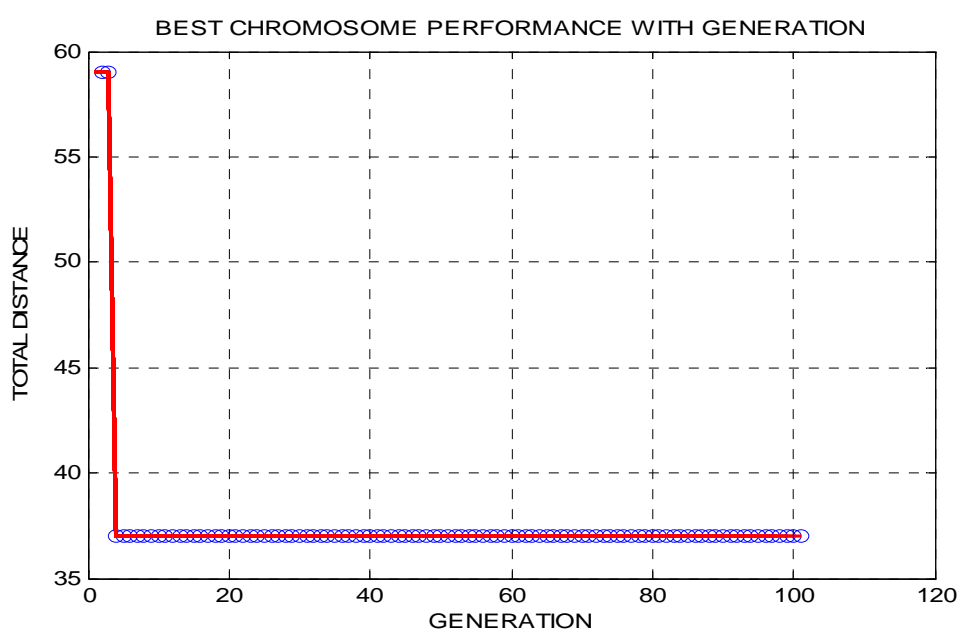

Fig-14 Experience agent adoptive genetic algorith, Connectivity path between 20[S] to 50[D] : [20126144 50 ]

Table I. Performance comparison for different algorithm under different WSN

\begin{tabular}{|c|c|c|c|c|}
\hline No. of sensors & \multicolumn{4}{|c|}{ Distance B/W Source to Destination nodes } \\
\hline & Static connectivity & RAGA & FAGA & EAGA \\
\hline 50 & 65 & 51 & 49 & $\mathbf{3 6}$ \\
\hline 100 & $\mathbf{4 3}$ & 53 & $\mathbf{4 3}$ & $\mathbf{4 3}$ \\
\hline 150 & 62 & 46 & $\mathbf{3 7}$ & $\mathbf{3 7}$ \\
\hline
\end{tabular}

It is observed with experiment that RAGA may take longer period to generate optimal solutions and there may be chance in between topology changes further which make the situation worst and obtained result are no way useful. If there is a high levels of change occur in topology it may difficult to find the optimal solutions within time span by FAGA.EAGA has shown the superior results in all cases in terms of high level of adaptive characteristics and faster exploration of global solution.this is possible because of exerience from past event help to handle the new event if it occurs.

\section{CONCLUSION}

Objective to increase the Connectivity of WSN can be obtained by concept of adaptive genetic algorithms. In this present paper a concept to increase connectivity of WSN by using adoptive genetic algorithm has presented, which help in find the solution to obtain high connectivity between source and destination. For various different sensors experiments has done on Redefine Adoptive genetic algorithm (RAGA), Flying Agents Adoptive genetic algorithm (FAGA), Experienced Adoptive genetic algorithm (EAGA) and shown very clearly that the proposed solution provide high connectivity between the source and destination by choosing minimum distance. In future the hybrid adoptive genetic algorithms is developed to enhance connectivity in dynamic environment of wireless sensor networks

\section{REFERENCES}

[1] Jennifer Yick, Biswanath Mukherjee, Dipak Ghosa, ”Wireless sensor network survey”, Compute Networks,Volume 52, Issue 12, 22 August 2008, Pages 2292-2330

[2] Amitabha Ghosh ,Sajal K. Das,” Coverage and connectivity issues in wireless sensor networks: A survey”, Pervasive and Mobile Computing,Volume 4, Issue 3, June 2008, Pages 303-334

[3] Mohamed Younis, Kemal Akkaya," Strategies and techniques for node placement in wireless sensor networks: A survey”, Ad Hoc Networks,Volume 6, Issue 4, June 2008, Pages 621-655

[4] Giuseppe Anastasi, Marco Conti, Mario Di Francesco , Andrea Passarella,” Energy conservation in wireless sensor networks: A survey”, Ad Hoc Networks, Volume 7, Issue 3, May 2009, Pages 537-568

[5] Nadjib Aitsaadi, Nadjib Achir, Khaled Boussetta, Guy Pujolle,’Artificial potential field approach in WSN deployment: Cost, QoM, connectivity, and lifetime constraints”, Computer Networks, Volume 55, Issue 1, 7 January 2011, Pages 84-105

[6] Lijuan Pu,, Wenming Cao,, XaoJiang Liang,”WSN Connected Coverage Analysis Based on Clifford Algebra,” Computer Science for Environmental Engineering and EcoInformatics,Volume 159 of the series Communications in Computer and Information Science pp 84-89,2011

[7] Raju Dutta, Shishir Gupta, Mukul K. Das ,’Power Consumption and Maximizing Network Lifetime During Communication of Sensor Node in WSN", Procedia Technology, Volume 4, 2012, Pages 158-162 
[8] Yun Wang ; Brendan M. Kelly ; Xiaolong Li,”On the network connectivity of wireless sensor networks following a random and nonuniform distribution”, Wireless and Mobile Computing, Networking and Communications (WiMob), 2013 IEEE 9th International Conference .

[9] Chuan Zhu, , Chunlin Zheng, , Lei Shu , Guangjie Han,” A survey on coverage and connectivity issues in wireless sensor networks”, Journal of Network and Computer Applications,Volume 35, Issue 2, March 2012, Pages 619-632.

[10] Soumyadip Sengupta, Swagatam Das, M.D. Nasir, B.K. Panigrahi,”Multi-objective node deployment in WSNs: In search of an optimal trade-off among coverage, lifetime, energy consumption, and connectivity”,Engineering Applications of Artificial Intelligence, Volume 26, Issue 1, January 2013, Pages 405-416

[11] Yun Wang , Yanping Zhang,, Jiangbo Liu,, Rahul Bhandari,” Coverage, Connectivity, and Deployment in Wireless Sensor Networks”, Recent Development in Wireless Sensor and Ad-hoc Networks, Signals and Communication Technology pp 25-44, December 2014

[12] R.Ramalakshmi,,S.Radhakrishnan,”Coverage and Connectivity Guaranteed Deterministic Deployment Pattern for WSN”, Computer Networks \& Communications (NetCom),Volume 131 of the series Lecture Notes in Electrical Engineering pp 341-347, 2015

[13] Chun-Wei Tsai, Pei-Wei Tsai, Jeng-Shyang Pan, Han-Chieh Chao,’Metaheuristics for the deployment problem of WSN: A review”, Microprocessors and Microsystems, Volume 39, Issue 8, November 2015, Pages 1305-1317

[14] Yanwen Wang, Hainan Chen, Xiaoling Wu, Lei Shu,”. An energy-efficient SDN based sleep scheduling algorithm for WSNs”,journal of Network and Computer Applications, Volume 59, January 2016, Pages 39-45.

[15] Sarika Yadav, Rama Shankar Yadav," A review on energy efficient protocols in wireless sensor networks”, Wireless Networks,January 2016, Volume 22, Issue 1, pp 335-350.

[16] Yatish K. Joshi, Mohamed Younis ,'Restoring connectivity in a resource constrained WSN”, Journal of Network and Computer Applications, Volume 66, May 2016, Pages 151-165.

\section{AUTHOR PROFILE}

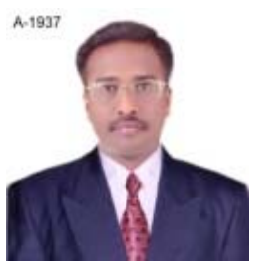

Mr. Channakrishnaraju has 19 years of teaching experience for UG and PG courses in computer Science and Engg and presently working as Associate Professor in the department of computer Science and Engg at Sri Siddhartha Institute of Technology, Tumkur. He Obtained B.E from Bangalore University in the year 1995, and PG in software systems in the year 2000. His research interests are in the areas Wireless sensor networks and Artificial Intelligence. Currently pursuing Doctoral degree in computer science \&Engg (wireless sensor networks) from Sri Siddhartha Academy of Higher Education,[SSAHE], Tumkur, under the guidance of Dr.M.Siddappa Professor and Head of the department of Computer Science and Engineering, Sri Siddhartha Institute of Technology, Tumkur. He published 19 Technical Papers in National, International Conference and journals.

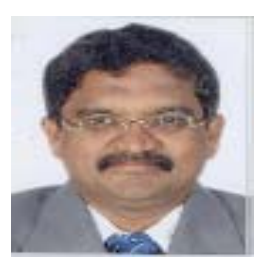

M.Siddappa received B.E degree in Computer Science \& Engineering from University of Mysore, Mysore, Karnataka, India in 1989, M.Tech from University of Mysore in 1993 and doctoral degree from Dr. MGR Educational Research Institute Chennai under supervision of Dr.A.S.Manjunatha, CEO, Manvish e-Tech Pvt. Ltd., Bangalore. He worked as project associate in IISc, Bangalore under Dr. M. P Srinivasan and Dr. V.Rajaraman (1991-93). He has teaching experience of 23 years and research of 10years. He published 35 Technical Papers in National, International Conference and Journals. He is a member of IEEE and Life member of ISTE. He is working in the field of data structure and algorithms, Artificial Intelligence, Image processing and Computer networking. He worked as Assistant Professor in Department of Computer Science \& Engineering from 1996 to 2003 in Sri Siddhartha Institute of Technology, Tumkur. Presently, he is working as Professor and Head, Department of Computer Science \& Engineering from 1999 to till date in Sri Siddhartha Institute of Technology, Tumkur. He has published nearly 65 papers in national and international journals. He is having citation index as 85 with j-index 4 and i10-index 3. He has received "Best Engineering teacher award" from ISTE for the year 2011 